\title{
Selecting Statistical Characteristics of Brain Signals to Detect Epileptic Seizures using Discrete Wavelet Transform and Perceptron Neural Network
}

\author{
Rezvan Abbasi ${ }^{1}$, and Mansour Esmaeilpour²* \\ ${ }^{1}$ Department of Computer System Architecture, Arak Branch, Islamic Azad University, Arak (Iran) \\ ${ }^{2}$ Department of Computer Engineering, Hamedan Branch, Islamic Azad University, Hamedan (Iran)
}

Received 26 June 2016 | Accepted 1 November 2016 | Published 23 December 2016

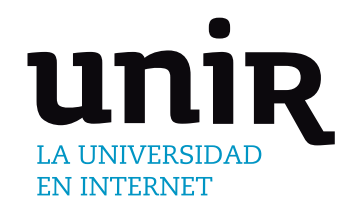

Electroencephalogram signals (EEG) have always been used in medical diagnosis. Evaluation of the statistical characteristics of EEG signals is actually the foundation of all brain signal processing methods. Since the correct prediction of disease status is of utmost importance, the goal is to use those models that have minimum error and maximum reliability. In anautomatic epileptic seizure detection system, we should be able to distinguish between EEG signals before, during and after seizure. Extracting useful characteristics from EEG data can greatly increase the classification accuracy. In this new approach, we first parse EEG signals to sub-bands in different categories with the help of discrete wavelet transform(DWT) and then we derive statistical characteristics such as maximum, minimum, average and standard deviation for each sub-band. A multilayer perceptron (MLP)neural network was used to assess the different scenarios of healthy and seizure among the collected signal sets. In order to assess the success and effectiveness of the proposed method, the confusion matrix was used and its accuracy was achieved98.33 percent. Due to the limitations and obstacles in analyzing EEG signals, the proposed method can greatly help professionals experimentally and visually in the classification and diagnosis of epileptic seizures.

\author{
Discrete Wavelet \\ Transforms \\ (DWT), Accuracy, \\ Electroencephalogram \\ Signals (EEG), \\ Multilayer Perceptron \\ (MLP), Epileptic \\ Seizure.
}

DOI: $10.9781 /$ ijimai.2017.456

\section{INTRODUCTION}

\begin{abstract}
- PILEPSY is one of the most prevalent neurological disorders among people[1]. It is estimated that 5 people are afflicted with epilepsy among each 1000 people. Epilepsy could be defined as a sudden change in the intracellular and extracellular potential difference. This definition implies that the type of neuron determines clinical demonstrations[2]. The automatic diagnosis of epileptic convulsions has attracted the attention of clinicians and engineers since 1970. The automatic prediction of seizures is useful in drug delivery systems and neural stimulation simulation devices $[3,4]$. An important issue in predicting epileptic convulsions is that they are predictable through analyzing the changes in the features of EEG signals that happen before the occurrence of seizures [5]. Epileptic seizures prediction needs further analysis due to the following reasons [6]:
\end{abstract}

1. Generally, their results are not repeatable. In other words, their confidence rate is not certain.

2. The dependence of the result on sensitivity and inaccurate prediction rate is not taken into account.

3. Their efficiency is not mostly acceptable and has a high acceptance and rejection rate.

* Corresponding author.

E-mail address: esmaeilpour@iauh.ac.ir
II. Materials and Methods

In an automatic epileptic convulsion detection system, a distinction should be made between the pre-convulsion, during convulsion, and post-convulsion EEG signals. Then, they should be analyzed [7]. Some studies focused on single-channel EEG signals, while some others focused on multi-channel recorded EEG signals [8]. This paper studied the epileptic and healthy signals of R. G. Andrzejak database from the University of Bonn [9]. The data relate to three different categories:normal situation of the patient, pre-seizure and seizure. The collected EEG signals include 5 categories which, respectively, are called (A,B,C,D,E).Each of these categories includes 100 singlechannel signals with a length of 26.3 seconds.

Category A: Surface EEG signal recorded from 5 healthy volunteers in a relaxed awake state with eyes open.

Category B: EEG signal recorded from 5 healthy volunteers in a relaxed state with eyes closed.

Category C: Deep signals recorded from epileptic patients during the interval between seizures from inside the area that caused the seizure. (focal signals)

Category D: Deep recorded signals from epileptic patients during the intervals between seizures from outside the area that caused the seizure. (non-focal signals)

Category E: Signals recorded from epileptic seizures.

All EEG signals were recorded with the 128-channel system with 
common average voltage. Sampling frequency in this database is $173.61 \mathrm{~Hz}$. According to the Nyquist Theorem ,the maximum useful sampling frequency is half of the sampling frequency. Here we have:

$$
\frac{173.61}{2}=86.6 \mathrm{~Hz}
$$

The placement design of surface electrodes is related to the 20-10 global system, is shown in Fig. 1. Therefore, the electrodes were named as follows $[10,11]$ :

FP1,FP2,F3,F4,C3,C4,P3,P4,F7,F8,T1,T2,T3,T5,T6,O1,O2,F2,P2

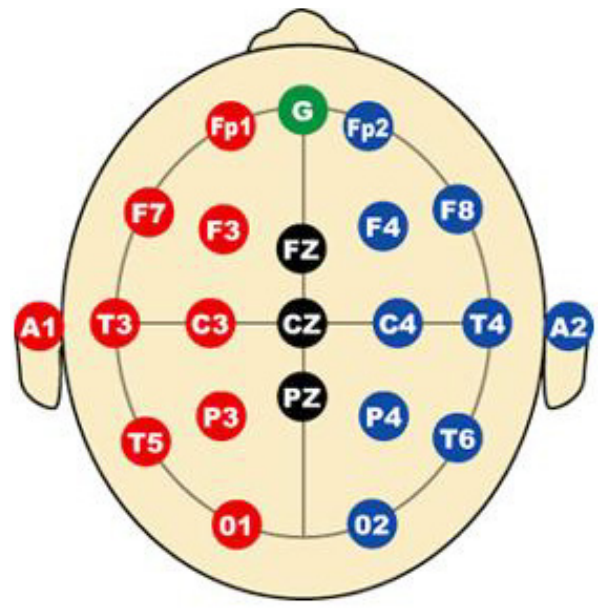

Fig. 1. The pattern of surface electrodes placement following that of the universal system 20-10.

The frontal lobe, temporal lobe, parietal lobe, central lobe, and occipital lobe were named F, T, P, C, and O, respectively [12].The Fig. 2 describes the anatomy of the brain with different regions[10].

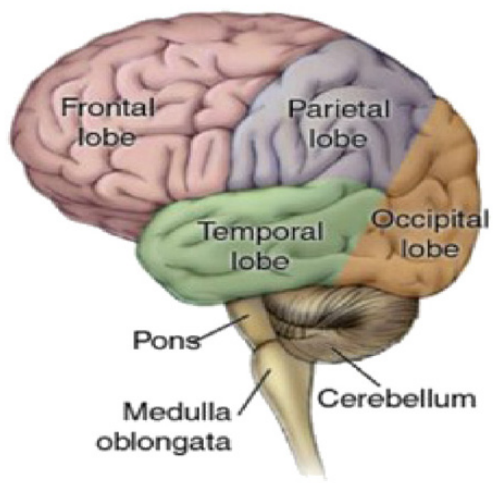

Fig. 2.Human brain structure

Fig. 3, 4 and 5 show healthy,convulsive and epileptic signals. The signal overlap healthy and epileptic shown in Fig. 6. In processing medical signals, it is vitally important to minimize existing noises and artifacts in order that they have the minimum effect on the feature extraction stage. In a wide-spreading spectrum, recorded EEG signals may contain technical and physiological noises [13]. By taking into account the physiological aspects, such as the artifacts caused by electrooculography (EOG), electromyography (EMG), and electrocardiography (ECG), and by applying an appropriate preprocessing, frequencies higher than $60 \mathrm{~Hz}$ were considered as noises and filtered.

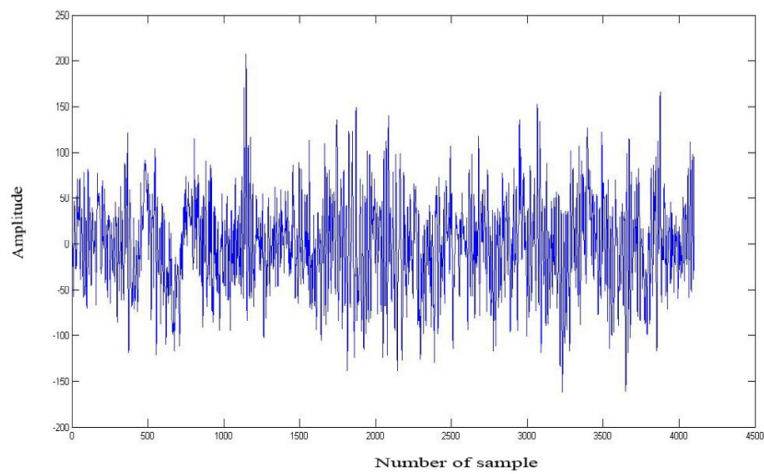

Fig. 3. An example of healthy signals.

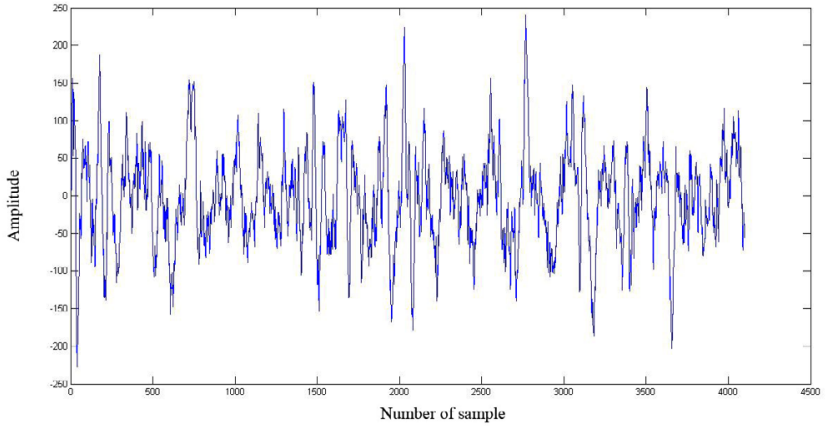

Fig. 4. An example of convulsive signals.

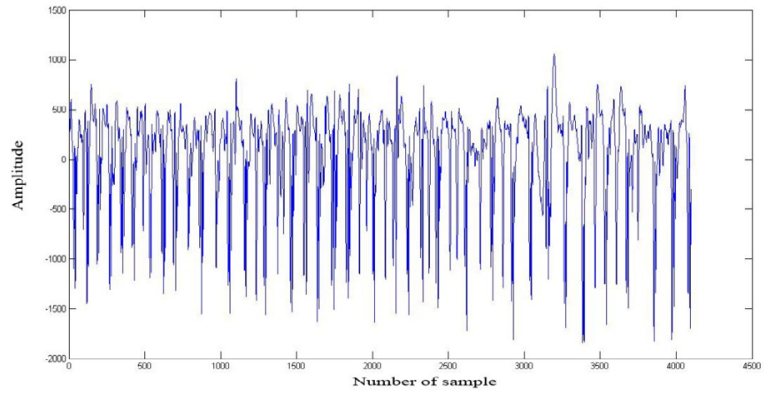

Fig. 5. An example of epileptic signals..

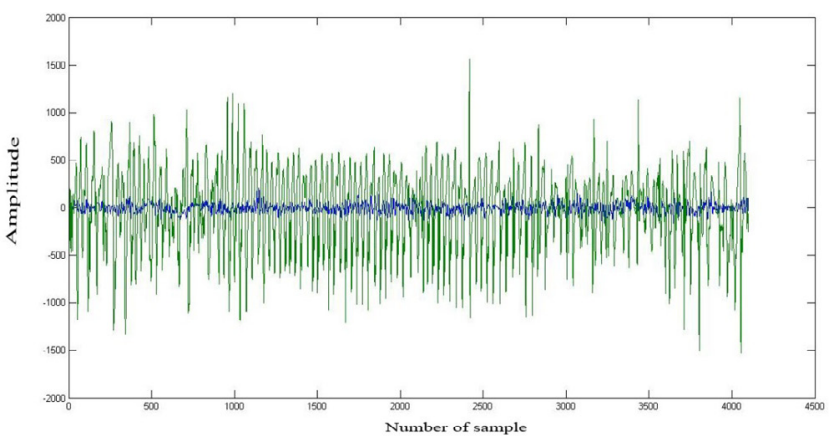

Fig. 6. Healthy and epileptic signals overlap rate.

\section{A. Features Extraction by DWT}

It is vitally important to select features which can best describe EEG signals for diagnosing convulsion and categorization. Since EEG signals are non-stationary waves [11], wavelet transform was used in their estimation. This frequency processing tool extracts a set of transient and local signals in space and frequency domains [14-16] . Wavelet transform decomposes signals into a set of basic functions called wavelet. These functions are obtained by applying delays, contractions, and transfer them on a unique function called wavelet pattern. Continuous wavelets are the functions resulted from an odd 
function using delays and transfers. They are dependent on transfer parameter. In order to remove noises and generate a signal appropriate for decomposition, EEG signals were limited by a low-pass filter and impulse response. Compared to EEG signals, sub-bands have more accurate information about neurons activities. They may not be evident in the original signals due to specific changes. Therefore, decomposition is carried out. The discrete wavelet signal is analyzed in the form of different frequency value bands and different magnifications. Using signal decomposition, the discrete wavelet signal is decomposed into coarse approximations and detailed information. In fact, discrete wave transform (DWT) employs a set of functions called measurement functions and wavelet functions. They are dependent on low-pass and high-pass filters. Decomposing signals into various frequency bands is simply achievable through successive applications of high-pass filters (HPFs) and low-pass filters (LPFs) [17, 18]. This decomposition method is known as multi-resolution decomposition. This type of analysis is illustrated in detail is shown in Fig. 7. The number of decomposition levels is selected based on dominant frequency components of the signal [17]. Selected levels maintain signal parts that highly correlate to the frequency related to signal classification in the wavelet.

The proposed method involves 4 layers and 5 frequency bands. It is due to the fact that higher order filters have fluctuations and lower order filters are rougher. Therefore, the signal was decomposed into D1-D4 details and the last estimation A4. Frequency sub-band values are shown in Table 1. Figures 8, 9, and 10 show the sub-bands resulted from the decomposition of healthy, convulsive, and epileptic signals using wavelet function Db4 in 4 levels. First, signals are decomposed into 5 levels. Then, level 5 approximation signal is removed. It has the lowest frequency band. It does not contain epileptic information, but contains noise information. Finally, the signal is reconstructed.

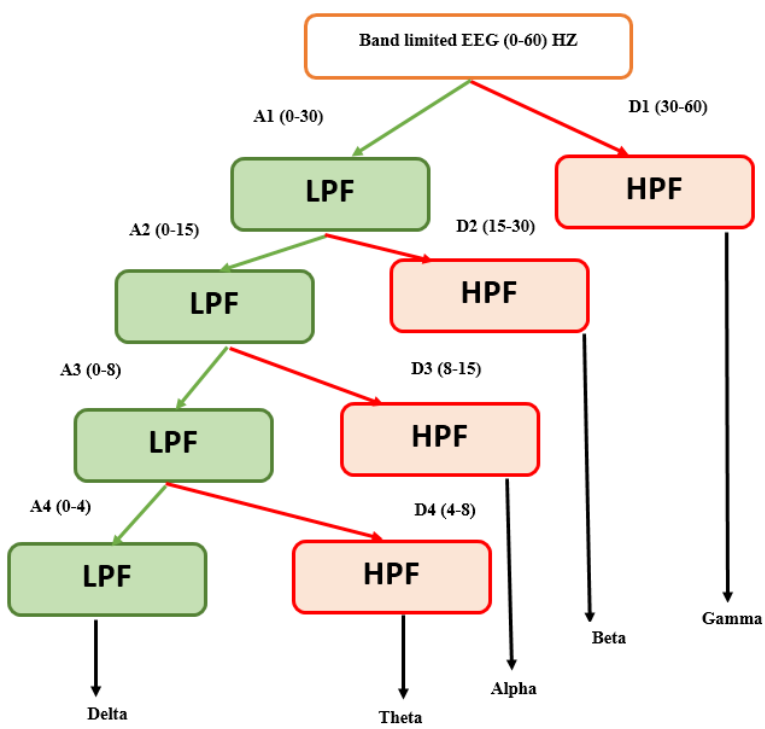

Fig. 7. Original signal decomposition level using daubechies wavelet transform.

TABLE I

WaVelet-Decomposition LeVel and EEG Sub-bands Relationship.

\begin{tabular}{cccc}
\hline $\begin{array}{c}\text { Band-limited } \\
\text { EEG }\end{array}$ & $\begin{array}{c}\text { Decomposition } \\
\text { level }\end{array}$ & Freqency band & $\begin{array}{c}\text { Frequency } \\
\text { bandwidth(Hz) }\end{array}$ \\
\hline$(0-4)$ & A4 & Delta & 4 \\
$(4-8)$ & D4 & Theta & 4 \\
$(8-15)$ & D3 & Alpha & 8 \\
$(15-30)$ & D2 & Beta & 15 \\
$(30-60)$ & D1 & Gamma & 30 \\
\hline
\end{tabular}

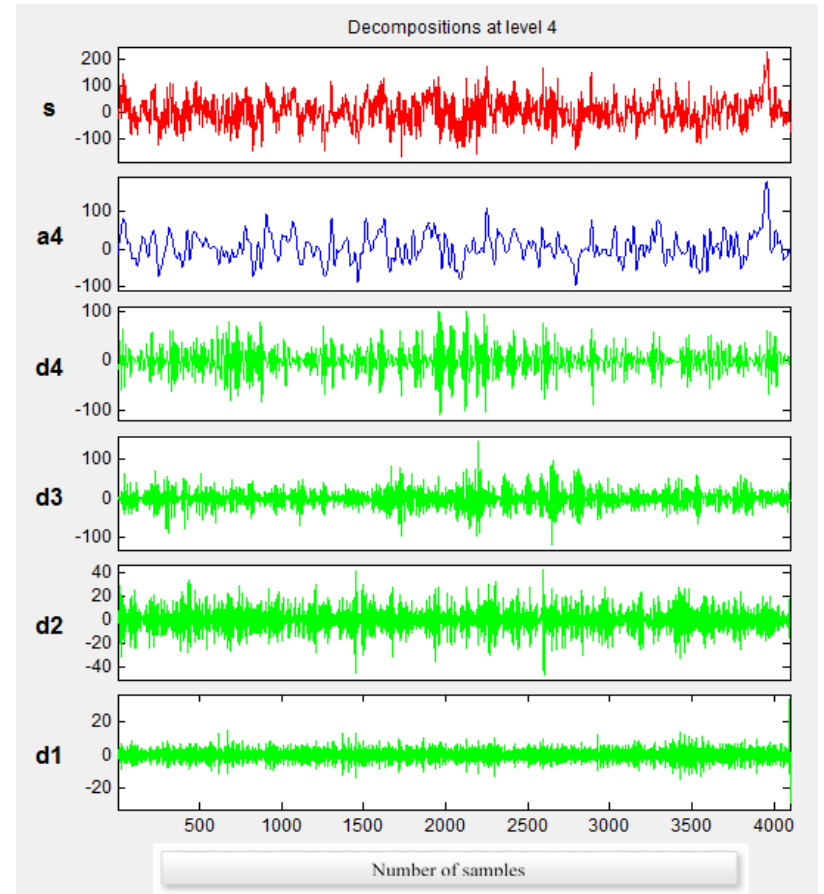

Fig. 8. A healthy signal with Daubechies 4 at level 4.

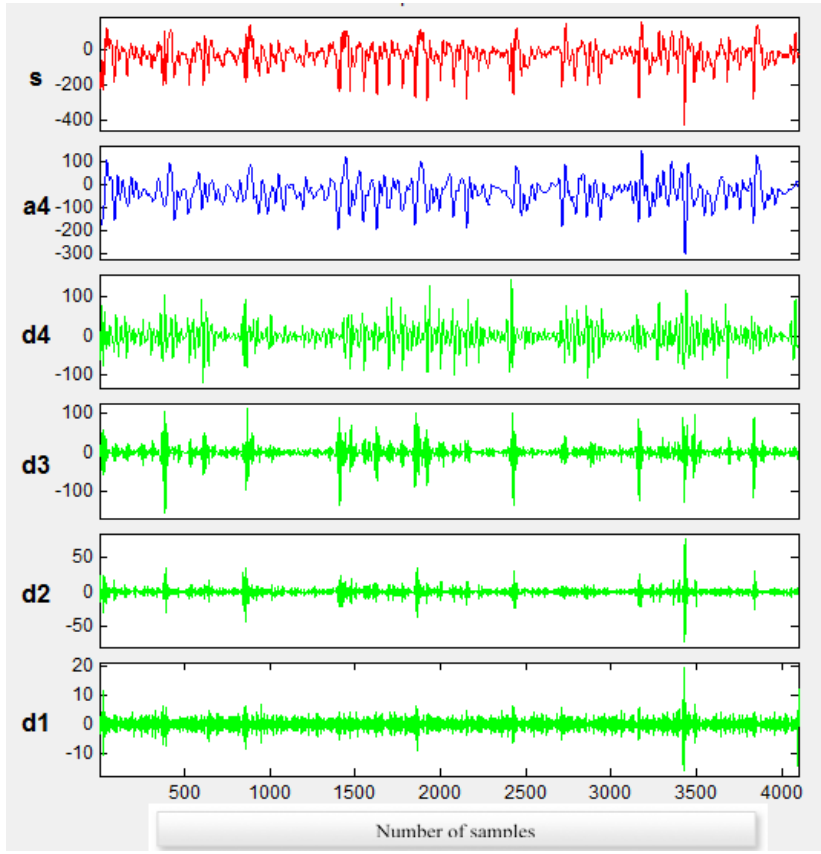

Fig. 9. A convulsive signal with Daubechies 4 at level 4.

Having applied pre-processing and carried out required processes, the desired feature vector was obtained. Statistical features, such as the maximum, minimum average, and standard deviation of each sub-band were used. Feature extracted are shown in Table 2. 


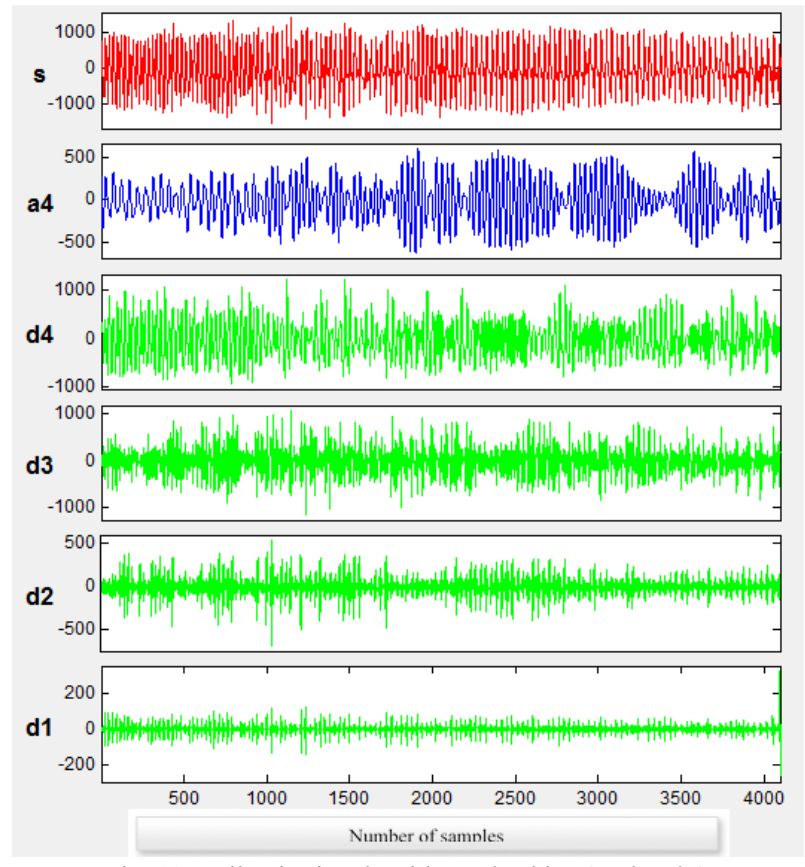

Fig. 10. Epileptic signals with Daubechies 4 at level 4.

\section{B. Classification by Neural Network}

Several statistical models have been proposed for classification and prediction. Classifying and predicting disorders based on risk factors is one of the applications of artificial neural networks [19,20]. Artificial neural networks are simply applicable to problems with no algorithmic solution, a complex algorithmic solution, and problems that are simple for people but difficult for computers [21]. They are also useful as an alternative solution for problems that generally have statistical solutions, such as regression modeling, predicting time series, cluster analysis, discriminate analysis, statistical decision-making problems, process control, and estimating the conditional distribution [19, 20]. An artificial perceptron multi-layer neural network [22] with error back propagation algorithm was used for evaluating different states of EEG signals, such as healthy, convulsive, and epileptic states. Structure of Multilayer perceptron shown in Fig. 11.

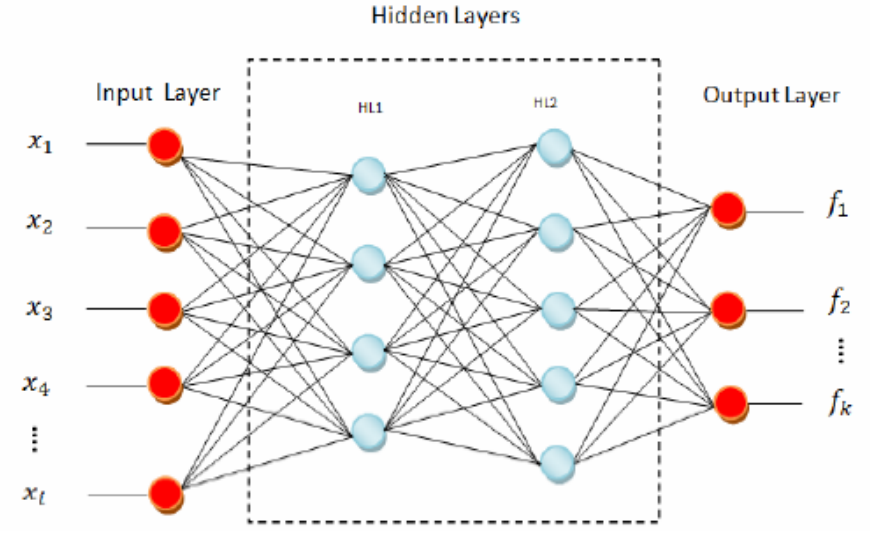

Fig. 11. Multilayer perceptron.

Having extracted desired statistical features using DWT, artificial neural network was used for classification. An artificial neural network with (12-15-3) structure and with sigmoid transfer function was designed and trained based on $80 \%$ of the available data. In the training phase, $80 \%$ of the collected data were used for training the artificial neural network. Having implemented the multi-layered perceptron (MLP) neural network using error back propagation learning (EBPL), having tested multiple layers and neurons, and having observed the errors, the most appropriate structure was selected. The most appropriate structure was (12-15-3), that is the network had four input variables for each category. The variables are the extracted statistical features, three output variables, and 15 neurons for maintaining the hidden layer. The output variable was defined based on three states, such as healthy, convulsive, and epileptic stages. Then, $20 \%$ of the available data were used for testing the neural network. In this phase, MLP with EBPL and (12-15-3) structure was used. For a more appropriate evaluation of results, feature and sensitivity were also calculated.

\section{Analyzing System Performance using Confusion Matrix}

Generally, in classification systems and disorder diagnosis systems, confusion matrix and receiving operating characteristic (ROC) curves are used for evaluating efficiency [23]. For analyzing the confusion matrix of classification and disorder diagnosis, four states are defined: true positive (TP), true negative (TN), false positive (FP), and false

TABLE II

Feature Extracted of Subbands

\begin{tabular}{|c|c|c|c|c|c|c|}
\hline \multirow{2}{*}{ Set } & \multirow{2}{*}{ Feature } & \multicolumn{5}{|c|}{ Subbands } \\
\hline & & D1 & D2 & D3 & D4 & A4 \\
\hline \multirow{4}{*}{ A } & Max & 12.039 & 31.306 & 75.769 & 120.014 & 192.677 \\
\hline & Min & -12.014 & -42.073 & -92.374 & -105.366 & -172.499 \\
\hline & Mean & -0.261 & 0.177 & 1.602 & 2.170 & 34.4130 \\
\hline & Std & 4.968 & 14.841 & 41.186 & 60.346 & 96.4623 \\
\hline \multirow{4}{*}{ B } & Max & 14.144 & 46.928 & 102.260 & 242.521 & 302.978 \\
\hline & Min & -14.757 & -51.484 & -139.186 & -157.733 & -208.899 \\
\hline & Mean & 0.472 & 0.089 & -7.327 & -2.741 & 24.0453 \\
\hline & Std & 6.048 & 17.938 & 60.054 & 88.495 & 146.456 \\
\hline \multirow{4}{*}{$\mathrm{C}$} & $\operatorname{Max}$ & 6.4079 & 17.195 & 49.523 & 142.374 & 231.600 \\
\hline & Min & -7.373 & -21.110 & -42.639 & -182.481 & -269.463 \\
\hline & Mean & 0.066 & -0.135 & 2.264 & -12.340 & -39.066 \\
\hline & Std & 2.800 & 9.514 & 25.913 & 95.077 & 153.392 \\
\hline \multirow{4}{*}{$\mathrm{D}$} & Max & 26.029 & 117.964 & 32.348 & 88.246 & 320.445 \\
\hline & Min & -20.682 & -82.160 & -61.542 & -89.151 & -175.767 \\
\hline & Mean & -0.193 & 0.112 & -2.211 & -2.636 & 94.158 \\
\hline & Std & 4.387 & 19.245 & 20.175 & 43.635 & 126.357 \\
\hline \multirow{4}{*}{ E } & Max & 258.080 & 644.365 & 1524.400 & 1420.100 & 1639.200 \\
\hline & Min & -325.450 & -1074.600 & -1508.900 & -1107.000 & -1917.600 \\
\hline & Mean & -0.133 & 0.105 & 65.561 & -77.229 & 281.401 \\
\hline & Std & 75.144 & 303.674 & 716.087 & 614.261 & 1138.500 \\
\hline
\end{tabular}


negative (FN) [24]. Each variable has a specific meaning in confusion matrix. TP is the number of patients suffering from epilepsy who are correctly diagnosed by the computer system. FP is the number of patients with epilepsy who are incorrectly diagnosed as healthy by the computer system. TN is the number of convulsive patients and healthy people correctly diagnosed as healthy by the computer system. FN is the number of convulsive patients or healthy people incorrectly diagnosed as epileptic by the computer system. $\mathrm{P}$ is the number of patients correctly classified by the system. In other words, it is the number of epileptic patients who are diagnosed correctly. It is also the number of healthy, convulsive, or non-epileptic people correctly classified. $\mathrm{N}$ is the number of the people who are incorrectly classified. In other words, it is the number of epileptic patients who are incorrectly diagnosed as healthy, or the number of healthy or convulsive people incorrectly diagnosed as epileptic or convulsive. Using the defined concepts, the efficiency of the proposed method was analyzed and they were named as sensitivity, specificity, classification, and precision, respectively. System precision is a measure that determines system's capability in diagnosing and classifying epileptic patients (true patients) correctly. Accuracy is but another index for evaluating such systems. It includes a more generalized perspective and domain of patient's classification systems. It is equal to the ratio of all correctly diagnosed cases, whether healthy or unhealthy, to all correctly or incorrectly classified cases [22], [25-28]. Sensitivity, Specificity, and Accuracy are defined as follows [10], [29, 30].

\section{RESULTS}

The following confusion matrix is obtained from applying the neural network on the test data. This set was a new one for the network and it was not trained by those data. Results show that the neural network worked correctly since healthy people and patients were correctly diagnosed. Predicting patients' condition based on the result in the training phase are shown in Table 3. Predicting patients' condition based on the result in the test phase are available in Table 4.

TABLE III

Predicting Patients' Condition based on the Result in the Training Phase

\begin{tabular}{ccccc}
\hline Total & Epileptic & Convulsive & Healthy & Predicting \\
\hline 60 & 0 & 0 & 60 & healthy \\
90 & 0 & 90 & 0 & convulsive \\
90 & 90 & 0 & 0 & epileptic \\
240 & 90 & 90 & 60 & Total \\
\hline
\end{tabular}

TABLE IV

Predicting Patients' Condition based on the Result in the Test Phase (20 Percent of the Samples, that is 60 Cases)

\begin{tabular}{ccccc}
\hline Total & Epileptic & Convulsive & Healthy & Predicting \\
\hline 8 & 0 & 0 & 8 & healthy \\
26 & 2 & 23 & 1 & convulsive \\
26 & 24 & 1 & 1 & epileptic \\
60 & 26 & 24 & 10 & Total \\
\hline
\end{tabular}

For a better understanding, it is necessary to calculate the sensitivity and specificity of the proposed method. According to confusion matrix and Equations (2), (3), and (4), sensitivity, specificity, and precision of the neural network are as follows. The proposed classification system's sensitivity is $100 \%$, which means the proposed system can diagnose all epileptic cases correctly. System's specificity was $97.1 \%$, which is significant. It means that the proposed system could diagnose $98.33 \%$ and even a higher number of the convulsive cases correctly. Results of confusion matrix for classification of test data are shown in Table 5.

$$
\text { Sensitivity }=\frac{T P}{T P+F N} \times 100
$$

$$
\begin{aligned}
& \text { Specificity }=\frac{T N}{F P+T N} \times 100 \\
& \text { Accuracy }=\frac{T P+T N}{T P+T N+F P+F N} \times 100
\end{aligned}
$$

TABLE V

Confusion Matrix (Accuracy, Sensitivity and Specificity) For Classification (Data Size: 240 Training Samples and 60 Testing Samples)

\begin{tabular}{ccc}
\hline Accuracy & Sensitivity & Specificity \\
\hline $98.33 \%$ & $100 \%$ & $97.1 \%$
\end{tabular}

\section{A. Comparison of the Proposed Method with other Methods}

Results from implementing the proposed MLP artificial neural network yield the highest sensitivity and precision. Many researchers have used wavelet transform in diagnosing epilepsy. Shoeb et al. used wavelet decomposition for generating feature vector [31]. Meier et al. exploited the combination of wavelet and time for extracting features as the input data for support vector machine (SVM) [32]. Abibullaevet all identified and presented various wavelet function for diagnosing convulsion and epilepsy, including ( bior1,3, Db5, Db2) [33]. Adeli et al. analyzed EEG signals for detecting EEG changes based on correlation function; frequency domain features, frequency time analysis, entropy, and wavelet transform [14]. Using chaos analysis, they divided the wavelets obtained from EEG signals into healthy and epileptic categories. Some other linear and non-linear methods were also used in predicting epileptic attacks [32], [34-38]. Results from various studies carried out using wavelet transform are shown in Table 6 [39]. Another disadvantage of existing solutions is their low precision and high dispersion which leads into a weak diagnosis. It is due to the high number of effective variables in physiological systems [6]. The aim of this study was to improve prediction results. Therefore, some changes were made to input and output variables. The type of selected wavelet function and variables were the reasons for a higher sensitivity and precision. Due to the limitation facing diagnosis systems, MLP structure was selected as the most appropriate artificial neural network structure with respect to the repetition of various conditions. The combination of artificial intelligence methods in classifying patterns, including artificial neural networks with wavelet transform resulted in an improved efficiency, agility, and diagnosis in the proposed method.

TABLE VI

Comparition Accuracy from previous Research Work [28].

\begin{tabular}{cc} 
Studies & Accuracy (\%) \\
Our Accuracy & 98.33 \\
Guler and Ubeyli(2005) & 97 \\
Kannathal et al.(2005) & 90 \\
Subasi(2007) & 95 \\
Chua et al(2008) & 88.78 \\
Ubeyli et al(2009) & 92.9 \\
Oweis and abdulahy(2011) & 94 \\
Orhan et al(2011) & 96.67 \\
Yuan et al(2011) & 96.5 \\
IV. ConCLUSION & \\
\hline
\end{tabular}

This paper aimed at proposing a new method for improving the precision of prediction and classifying different states of EEG signals into healthy, convulsive, and epileptic states. Using wavelet transform and MLP, sensitivity, specificity, and precision indexes were improved significantly. 


\section{REFERENCES}

[1] Litt B Echauz, J. Prediction of epileptic seizures. The Lancet Neurology 2002;1: 22-30.

[2] Subasi A, Erçelebi E. Classification of EEG signals using neural network and logistic regression. Computer methods and programs in biomedicine 2005;78:87-99.

[3] Stein A.G, Eder H. G, Blum D. E, Drachev A, Fisher R. S. An automated drug delivery system for focal epilepsy. Epilepsy research 2000;39: 103-114.

[4] Osorio I, Frei M. G. Real-time detection, quantification, warning, and control of epileptic seizures: The foundations for a scientific epileptology. Epilepsy \&Behavior 2009;16: 391-396.

[5] Mormann F, Kreuz T, Andrzejak R. G, David P,Lehnertz K, Elger C. E. Epileptic seizures are preceded by a decrease in synchronization. Epilepsy research 2003;53: 173-185.

[6] Iasemidis L. D. Epileptic seizure prediction and control. In: IEEE 2003 Biomedical Engineering; pp. 549-558.

[7] Tong S, Thakor N. V. Quantitative EEG analysis methods and clinical applications. Artech House, 2009.

[8] Deburchgraeve W,Cherian P. J, De Vos M, Swarte R. M, Blok J. H, Visser G. H, Van Huffel S. Automated neonatal seizure detection mimicking a human observer reading EEG. Clinical Neurophysiology 2008;119: 2447-2454

[9] Andrzejak R. G, Lehnertz K, Mormann F, Rieke C, David P, Elger C. E. Indications of nonlinear deterministic and finite-dimensional structures in time series of brain electrical activity: Dependence on recording region and brain state. Physical Review E 2001;64: 061907.

[10] Kumari, Pinki, and AbhishekVaish. "Brainwave based user identification system: A pilot study in robotics environment." Robotics and Autonomous Systems 65 (2015): 15-23.

[11] Kumari, Pinki, and AbhishekVaish. "Feature-level fusion of mental task's brain signal for an efficient identification system." Neural Computing and Applications: 1-11.

[12] Durka P. J. Adaptive time-frequency parametrization of epileptic spikes. Physical Review E 2004;69:051914.

[13] Sörnmo L, Laguna P. Bioelectrical signal processing in cardiac and neurological applications. Academic Press 2005.

[14] Adeli H, Ghosh-Dastidar S, Dadmehr N. A wavelet-chaos methodology for analysis of EEGs and EEG subbands to detect seizure and epilepsy.In: IEEE 2007 Biomedical Engineering; 54: 205-211.

[15] Guo L, Rivero D, Dorado J, Rabunal J. R, Pazos A. Automatic epileptic seizure detection in EEGs based on line length feature and artificial neural networks. . Journal of neuroscience methods 2010; 191: 101-109.

[16] Yuan Q, Zhou W, Li S, Cai D. Epileptic EEG classification based on extreme learning machine and nonlinear features. Epilepsy research 2011; 96: 29-38.

[17] Subasi A. Epileptic seizure detection using dynamic wavelet network. Expert Systems with Applications 2005;29: 343-355.

[18] Khan Y. U, Farooq O, Sharma P. Automatic detection of seizure onset in pediatric EEG. International Joural of Embeded Systems and Applications 2012; 2: 81-89.

[19] Livingstone D. J. Artificial Neural Networks: Methods and Applications (Methods in Molecular Biology). Humana Press, 2008.

[20] Dreiseitl S, Ohno-Machado L. Logistic regression and artificial neural network classification models: a methodology review. Journal of biomedical informatics 2002;35: 352-359.

[21] Zini G, d'Onofrio G. Neural network in hematopoietic malignancies. Clinica chimica acta 2003; 333:195-201.

[22] Ramchoun H, Amine M, Idrissi J, Ghanou Y, Ettaouil M. Multilayer Perceptron: Architecture Optimization and Training. International Journal of Interactive Multimedia and Artificial Inteligence. 2016;4(Special Issue on Artificial Intelligence Underpinning).

[23] Semwal, Vijay Bhaskar, Manish Raj, and Gora Chand Nandi. "Biometric gait identification based on a multilayer perceptron." Robotics and Autonomous Systems 65 (2015): 65-75.

[24] Abhinav-Vishwa MK, Lal SD, Vardwaj P. Clasification of arrhythmic ECG data using machine learning techniques. International Journal of Interactive Multimedia and Artificial Intelligence. 2011 Dec;1(4).

[25] Vaish, Abhishek, and PinkiKumari. "A Comparative Study on Machine Learning Algorithms in Emotion State Recognition Using
ECG." Proceedings of the Second International Conference on Soft Computing for Problem Solving (SocProS 2012), December 28-30, 2012. Springer India, 2014.

[26] Semwal, Vijay Bhaskar, KaushikMondal, and G. C. Nandi. "Robust and accurate feature selection for humanoid push recovery and classification: deep learning approach." Neural Computing and Applications: 1-10.

[27] Singha, Joyeeta, and RabulHussainLaskar. "Self co-articulation detection and trajectory guided recognition for dynamic hand gestures." IET Computer Vision (2015).

[28] Singha, Joyeeta, and RabulHussainLaskar,'Hand gesture recognition using two-level speed normalization, feature selection and classifier fusion”, Multimedia Systems, 2016.

[29] Kumari, Pinki, and AbhishekVaish. "Information-Theoretic Measures on Intrinsic Mode Function for the Individual Identification Using EEG Sensors."Sensors Journal, IEEE 15.9 (2015): 4950-4960.

[30] Singha, Joyeeta, and RabulHussainLaskar, "Recognition of global hand gestures using self co-articulation information and classifier fusion", Journal on Multimodal User Interfaces, Volume 10, Issue 1, pp 77-93.

[31] Shoeb A, Edwards H, Connolly J, Bourgeois B, Treves S. T, Guttag J. Patient-specific seizure onset detection. Epilepsy \& Behavior 2004; 5:483498.

[32] Meier R, Dittrich H, Schulze-Bonhage A, Aertsen A. Detecting epileptic seizures in long-term human EEG: a new approach to automatic online and real-time detection and classification of polymorphic seizure patterns. Journal of Clinical Neurophysiology 2008; 25: 119-131.

[33] Abibullaev B, Kim M. S, Seo H. D. Seizure detection in temporal lobe epileptic EEGs using the best basis wavelet functions. Journal of medical systems 2010; 34:755-765.

[34] Kannathal N, Choo M. L, Acharya U. R, Sadasivan P. K. Entropies for detection of epilepsy in EEG. Computer methods and programs in biomedicine 2005; 80: 187-194.

[35] Polat K, Güneş S. Classification of epileptiform EEG using a hybrid system based on decision tree classifier and fast Fourier transform. Applied Mathematics and Computation 2007;187: 1017-1026.

[36] Chan A. M, Sun F. T, Boto E. H, Wingeier B. M. Automated seizure onset detection for accurate onset time determination in intracranial EEG. Clinical Neurophysiology 2008; 119:2687-2696.

[37] Aarabi A, Fazel-Rezai R, Aghakhani Y.A fuzzy rule-based system for epileptic seizure detection in intracranial EEG. Clinical Neurophysiology 2009; 120: 1648-1657.

[38] Niederhauser J. J, Esteller R, Echauz J, Vachtsevanos G, Litt B. Detection of seizure precursors from depth-EEG using a sign periodogram transform .In: IEEE 2003 Biomedical Engineering; 50: 449-458.

[39] Orosco L,Correa A.G,Laciar E.Review:Asurvey of Performance and Techniques for Automatic Eplilepsy Detection. Journal of Medical and Biological Engineering 2013;33:526-537.

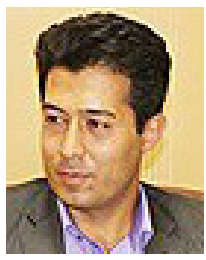

\section{Mansour Esmaeilpour}

Mansour Esmaeilpour, received the B.S. and M.S. degrees in Computer Engineering-Software Engineering in Iran, in 2002 and 2004, respectively. He also received the Ph.D. degree in Computer Engineering from The National University of Malaysia, in 2012. Currently, he is an assistant professor in Computer Engineering Department at Islamic Azad University, Hamedan Branch, Hamedan, Iran. As well as, he worked from 2013 to 2014 as Postdoctoral Researcher at National University of Malaysia. His research interests include Data mining, Process Mining, Learning Systems and Machine Learning.

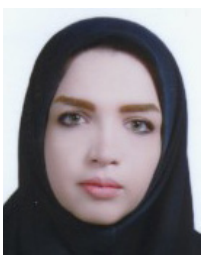

Rezvan Abbasi

Rezvan Abbasi received her B.S in Computer Software Engineering and M.S in Computer System Architecture Engineering from Islamic Azad University, Arak Branch, Arak and she is PhD student in Computer System Architecture Engineering in Islamic Azad University, Kerman branch now. Her main research interest is in computer arithmetic, signal processing and Nano-technology Design. 